\title{
Out-patient psychotherapy for borderline personality disorder: cost-effectiveness of schema-focused therapy $v$. transference-focused psychotherapy
}

Antoinette D. I. van Asselt, Carmen D. Dirksen, Arnoud Arntz, Josephine H. Giesen-Bloo, Richard van Dyck, Philip Spinhoven, Willem van Tilburg, Ismay P. Kremers, Marjon Nadort and Johan L. Severens

\section{Background}

Schema-focused therapy (SFT) and transference-focused psychotherapy (TFP) for borderline personality disorder were recently compared in a randomised multicentre trial.

\section{Aims}

To assess the societal cost-effectiveness of SFT $v$. TFP in treating borderline personality disorder.

\section{Method}

Costs were assessed by interview. Health-related quality of life was measured using EQ-5D. Outcomes were costs per recovered patient (recovery assessed with the Borderline Personality Disorder Severity Index) and costs per qualityadjusted life-year (QALY).

\section{Results}

Mean 4-year bootstrapped costs were €37826 for SFT and
$€ 46795$ for TFP (95\% uncertainty interval for difference -21775 to 3546); QALYS were 2.15 for SFT and 2.27 for TFP (95\% UI -0.51 to 0.28 ). The percentages of patients who recovered were $52 \%$ and $29 \%$ respectively. The SFT intervention was less costly and more effective than TFP (dominant), for recovery; it saved €90 457 for one QALY loss.

\section{Conclusions}

Despite the initial slight disadvantage in QALYS, there is a high probability that compared with TFP, SFT is a cost-effective treatment for borderline personality disorder.

\section{Declaration of interest}

None. Funding detailed in Acknowledgements.
Borderline personality disorder is well known as a severe psychiatric condition. The prevalence of this disorder is estimated at $1-2.5 \%$ in the worldwide population, ${ }^{1}$ and at $10-50 \%$ among psychiatric patients. Societal costs associated with borderline personality disorder are substantial. ${ }^{2}$ Recently, the effectiveness of two out-patient psychotherapies - schema-focused therapy (SFT) and transference-focused psychotherapy (TFP) - was compared. ${ }^{3}$ Both treatments aim to achieve full recovery from the disorder, unlike other therapies. ${ }^{4}$ In that comparison both forms of therapy succeeded in reducing disorder-specific and general psychopathologic dysfunction, and improving health-related quality of life, with SFT being more effective on all measures. However, the most effective treatment is not necessarily the most cost-effective treatment. In the context of healthcare budget constraints, an economic evaluation can inform decisions concerning which healthcare services to offer to patients. Therefore, a cost-effectiveness analysis was performed comparing these two forms of therapy.

\section{Method}

\section{Patients and assessments}

In a multicentre trial in The Netherlands, 86 patients from four study locations were randomly allocated to either SFT $(n=44)$ or TFP $(n=42)$. A pre-randomisation assessment was performed; subsequently, 3-monthly assessments were made for 3 years, with a final follow-up assessment 4 years after the baseline interviews. Both interventions were individual therapies consisting of 50 min sessions twice a week for 3 years. Central to SFT is the assumption of four schema modes specific to borderline personality disorder; recovery is achieved when dysfunctional schemas no longer control or rule the patient's life. Central to TFP is a negotiated treatment contract between patient and therapist, being the treatment frame; recovery is reached when good and bad representations of self (and others) are integrated and when fixed, primitive internalised object relations are resolved. Twelve participants in the SFT group and 22 in the TFP group left the study early. A further 6 (14\%) participants in the SFT group and $2(5 \%)$ in the TFP group successfully (according to therapist and patient) terminated treatment within 3 years. For details, see Giesen-Bloo et al. $^{3}$

\section{Resources used}

At every assessment a structured cost interview was administered by an independent research assistant. Formal registries such as hospital information systems or insurer's databases are considered to be incomplete, since a considerable amount of resource use is situated outside (mental) healthcare institutions. ${ }^{5}$ Besides, individual patient data cannot be traced from registries. Therefore, patient-reported prospective cost diaries, ${ }^{6}$ or retrospective cost interviews, ${ }^{7}$ are the preferred instruments covering all relevant events. We chose a 3 -month recall interview, ${ }^{8}$ since a prospective cost diary was expected to lead to more missing items, given the patient characteristics. From a societal perspective, the cost interview covered work status and absence, sources of income, domestic activities, informal care, medication use, alcohol and drugs, out-of-pocket expenses, and consumption of healthcare and societal resources (including visits to general practitioners, 
hospitals, psychiatrists and psychologists, crisis centres, alternative healers, social work and drug rehabilitation centres). Informal care is an important item in mental healthcare, since people with mental illness can impose a heavy burden on their environment: those close to the patient (family, friends or neighbours) take care of the patient and take over domestic tasks. A key characteristic of informal care is that caregivers would not want to care for someone outside their social environment for a similar wage. ${ }^{9}$ Out-of-pocket costs are actual expenses reported by the individual and are highly relevant in people with borderline personality disorder. These costs relate to (for instance) excessive smoking and shopping, binge eating and extremely high telephone bills. ${ }^{5}$

The cost interview (based on the cost diary described by Goossens et al) ${ }^{6}$ was judged by various experts and pilot tested by research assistants in patients, after which both face validity and content validity of the interview were improved. The questions constituting the cost interview were read to the participants, who indicated whether or not they had been absent from work, used a particular healthcare facility, etc. Only costs related to the person's personality disorder were considered relevant, because unrelated costs were not expected to differ between the treatment groups. Patients were explicitly asked whether they thought resource use was linked to problems arising from their personality disorder. In the rare cases in which resource use was probably disorder-related but the patient indicated that it was not, patients' judgements were overruled. For instance, if patients had regular contact with social services because of problems with raising their children and they rated this as not disorder-related, the contacts were still included in the analysis. Since for alcohol and drugs it is difficult to decide which part of the cost is related to the personality disorder, all alcohol and drug expenses were considered to be disorder-related.

The number of therapy sessions was recorded by the therapist. The number, duration and time (inside or outside office hours) of telephone contacts with the therapist were extracted from the standardised session form therapists filled out after each session. Training and supervision costs were not taken into account, because they are training costs and as such not part of the therapy.

\section{Unit prices}

Standardised Dutch unit prices were used. ${ }^{10}$ When a standardised unit price was not available, prices were based on tariffs. Medication costs were obtained from the Dutch Pharmacotherapeutic Compass. ${ }^{11}$ Productivity costs, incurred when patients are unable to perform paid work, were calculated according to the human capital approach, ${ }^{12}$ in which the number of hours of absence from work was multiplied by the actual gross wage per hour. For unpaid work and study, the number of hours absent was multiplied by a shadow price. The shadow price was also applied to the informal care. All unit prices were expressed in euros for the year 2000 .

\section{Outcome measures}

Effectiveness was expressed as the proportion of recovered patients according to the Borderline Personality Disorder Severity Index (BPDSI) version $\mathrm{IV}^{3,13}$ a 70 -item index with nine dimensions, representing the DSM-IV borderline personality disorder criteria. Total scores range from 0 to 90 . When the BPDSI score was below 15 at follow-up, patients were considered to have recovered. Additionally, effectiveness was expressed as quality-adjusted lifeyears (QALYs). A QALY is a measure of life expectancy, weighted by the health-related quality of life (HRQoL) represented by utility scores. Health-related quality of life was measured with the
EQ-5D, which contains five dimensions: mobility, self-care, daily activities, pain/discomfort, and depression/anxiety. ${ }^{14}$ Each dimension is rated at three levels: no problem, some problems and major problems. Based on preferences elicited from a general UK population, EQ-5D health states can be converted into utility scores. ${ }^{15}$ The maximum possible number of QALYs within 4 years is 4 ( 4 years multiplied by 1 , the optimal health state).

\section{Cost-effectiveness}

Two incremental cost-effectiveness ratios (ICERs) were determined. These ratios are calculated by dividing the difference in costs between the treatments by the difference in effectiveness between the treatments, and represents the extra amount of money that has to be invested or will be saved to gain or lose one extra unit of effect. The first ICER was the cost per recovered patient and the second ICER was the cost per QALY gained.

\section{Data analysis}

Costs and effects were discounted at $4 \% .{ }^{10}$ Missing items in otherwise completed assessments were imputed with SPSS (version 12 for Windows) Missing Value Analysis, option regression. Completely missing assessments because of patients who left the study or completed the treatment before the 3-year limit were analysed by carrying the last observation forward, in accordance with GiesenBloo et al. ${ }^{3}$

For BPDSI recovery status results after 4 years, a logistic regression was performed with treatment group and baseline BPDSI score as covariates, again in accordance with Giesen-Bloo (further details available from the authors). Since cost data are generally highly skewed, and not distributed normally, bootstrap simulations with 1000 replications were performed to estimate uncertainty intervals around the mean costs. ${ }^{16,17}$ For the same reasons, QALY scores and both ICERs were bootstrapped with 1000 replications. The uncertainty interval is represented by the 2.5 th and 97.5th percentiles. Results of ICER bootstraps are presented in cost-effectiveness planes and cost-effectiveness acceptability curves (CEACs). Cost-effectiveness planes show differences in costs on the vertical axis and differences in effect on the horizontal axis. Bootstrapped cost-effectiveness pairs located in the northwest quadrant indicate SFT to be inferior to TFP (more costly and less effective than TFP). Cost-effectiveness pairs located in the south-east quadrant show SFT to be dominant over TFP (more effective and less costly than TFP). With respect to the other two quadrants (higher costs for better effectiveness and lower costs for lower effectiveness) the choice of an intervention depends on the threshold value, i.e. what society is prepared to pay for an effectiveness gain, or willing to accept as savings for effectiveness loss. The CEAC represents the probability that, given a certain threshold for the willingness to pay for a QALY or for recovering a patient, the intervention is cost-effective. ${ }^{18}$

\section{Secondary analyses}

Secondary analyses were performed to assess robustness of the results, initially using the last observation carried forward method for patients who completed the therapy early with consent of the therapist but did not complete later assessments, and imputing baseline values for true 'drop-outs', i.e. patients who stopped therapy without consent of the therapist. We then conducted a 'completers' analysis, analysing data for only those patients who completed treatment. Patients who completed early with consent of their therapist were included in this analysis, those who dropped out were not. 
As costs at baseline were not comparable between both groups, a correction was performed for the following measurements by means of a regression analysis as recommended by Manca et al. ${ }^{19} \mathrm{~A}$ correction was also performed for utility and BPDSI scores. An analysis per recovered patient with a healthcare perspective, meaning that only direct healthcare costs (including costs of therapy) were considered, was followed by an investigation of the impact of the unit price of a therapy session, the unit price of informal care and out-of-pocket costs on total costs. We separately doubled and halved the costs of these items. The results of the first four secondary analyses are again represented in a CEAC, combined with the CEAC of the main analysis.

\section{Results}

The demographic characteristics of the participants are shown in Table 1.

\section{Costs}

\section{Baseline assessment}

At baseline, costs were not distributed normally ( $P=0.029$, Kolmogorov-Smirnov test. The bootstrapped total costs at baseline were $€ 4324$ for TFP and $€ 3331$ for SFT, a difference of about $€ 900$. However, neither of the cost categories nor the total societal cost was significantly different between conditions.

\section{After start of treatment}

Bootstrapped societal costs over 4 years were $€ 46795$ for TFP and $€ 37826$ for SFT (non-significant difference; Table 2). The direct non-healthcare costs were significantly different between the two intervention therapies, mainly owing to informal care costs. With respect to direct healthcare costs, treatment costs (including costs of telephone contacts with the therapist for SFT) were about $€ 2200$ higher for SFT, whereas the costs of visits to mental healthcare centres and other healthcare facilities were about $€ 3500$ higher for TFP. Table 3 presents mean societal costs, EQ-5D utility scores, BPDSI scores and percentage recovered patients per measurement. Costs in both groups decreased with time. In addition, aside from the second assessment when costs were comparable between the two groups, the TFP costs were continuously higher.

\section{Effectiveness}

\section{BPDSI score}

The proportion of patients who had recovered after 4 years was $52 \%$ for the SFT group and $29 \%$ for the TFP group (Table 3). Logistic regression analysis with treatment group and BPDSI baseline score as covariates showed a significant effect in favour of SFT (Wald test 4.45, $P=0.035$; OR $=2.64,95 \%$ CI 1.07-6.49). Without the baseline BPDSI the group effect was similar. (For details of the BPDSI results, see Giesen-Bloo et al; ${ }^{3}$ further details are available from the authors.)

\section{Quality-adjusted life-years}

For one participant no EQ-5D score was available, therefore QALY results and ICERs for cost per QALY are based on 85 patients. Table 3 shows that baseline utility scores were not distributed normally $(P=0.037$, Kolmogorov-Smirnov test). Baseline utility scores were 0.49 for the TFP group and 0.46 for the SFT group ( $P=0.734$, Mann-Whitney test). In TFP, utility scores increased early in treatment and more or less remained stable, whereas in SFT utility scores continued to increase. As a result, total QALYs over the 4-year period were 2.15 for SFT and 2.27 for TFP. The bootstrapped difference in QALYs between SFT and TFP of -0.12 (95\% UI -0.51 to 0.28 ) was not statistically significant.

\section{Cost-effectiveness}

\section{Societal costs per recovered patient}

As the societal costs in the SFT group were lower and recovery rate was higher compared with the TFP group, SFT was dominant over TFP (Table 4). The bootstrapped results (Fig. 1) show that $91 \%$ of the cost-effectiveness pairs are in the south-east quadrant, where SFT is dominant. Another $8 \%$ lie in the north-east (i.e. more costly, more effective) quadrant, and $1 \%$ is located in both west quadrants, where SFT is less effective than TFP. The CEAC (Fig. 2, main analysis) indicates that, regardless of the threshold value, the chance that SFT is cost-effective is over $90 \%$.

\section{Societal cost per QALY}

The incremental cost-effectiveness ratio based on the QALY shows that $€ 90457$ is saved when one QALY is sacrificed, as SFT was less costly and less effective than TFP (Table 4 ). The bootstrapped results (Fig. 3) show that $68 \%$ of the cost-effectiveness pairs are in the south-west quadrant, where SFT is less costly

Table 1 Baseline characteristics of study participants $(n=86)$

$\left.\begin{array}{|lcc|}\hline & \text { SFT group }(n=44) & \text { TFP group ( } n=42) \\ \hline \text { Age, years: mean (s.d.) } & 31.70(8.9) & 29.45(6.5) \\ \hline \text { Gender } & & \\ \quad \text { Female, } n \text { (\%) } & 40(90.9) & 40(95.2) \\ \hline \text { Education, } n \text { (\%) } & & 0.15 \\ \quad \text { Graduate/professional } & 6(13.6) & 4(9.5) \\ \text { College graduate } & 3(6.8) & 7(16.7) \\ \text { Some college } & 17(38.6) & 14(33.3) \\ \text { High-school graduate } & 5(11.4) & 10(23.8) \\ \text { Grades 7-11 } & 13(29.6) & 7(16.7)\end{array}\right\}$




\begin{tabular}{|c|c|c|c|c|}
\hline Cost category & TFP costs, $€$ & SFT costs, $€$ & Incremental costs, $€$ & 2.5-97.5 percentile \\
\hline \multicolumn{5}{|l|}{ Direct healthcare mean costs } \\
\hline Psychotherapy & 10876 & 12946 & & \\
\hline Contact with therapist by telephone & 2 & 132 & & \\
\hline Other care at treatment centre & 875 & 565 & & \\
\hline General practitioner & 191 & 112 & & \\
\hline Healthcare, other ${ }^{a}$ & 6883 & 3851 & & \\
\hline Medication & 1494 & 1273 & & \\
\hline Alternative healers & 190 & 91 & & \\
\hline Subtotal & 20512 & 18969 & & \\
\hline Bootstrapped subtotal, mean (s.d.) & 20589 (2754) & $19020(2097)$ & -1269 & -8514 to 4973 \\
\hline \multicolumn{5}{|l|}{ Direct non-healthcare mean costs } \\
\hline Paid help & 255 & 170 & & \\
\hline Societal services ${ }^{\mathrm{b}}$ & 353 & 238 & & \\
\hline Informal care & 8223 & 3053 & & \\
\hline Subtotal & 8831 & 3460 & & \\
\hline Bootstrapped subtotal, mean (s.d.) & 8915 (2189) & $3482(785)$ & -5433 & -10385 to 1469 \\
\hline \multicolumn{5}{|l|}{ Lost production mean costs } \\
\hline Paid work & 890 & 2016 & & \\
\hline Unpaid work & 3612 & 536 & & \\
\hline Domestic tasks & 7107 & 8255 & & \\
\hline Subtotal & 11609 & 10807 & & \\
\hline Bootstrapped subtotal, mean (s.d.) & $11495(2886)$ & 10704 (2394) & -791 & -8204 to 6443 \\
\hline \multicolumn{5}{|l|}{ Out-of-pocket mean costs } \\
\hline Reported by patient ${ }^{c}$ & 2821 & 1953 & & \\
\hline Alcohol and drugs & 3238 & 2804 & & \\
\hline Subtotal & 6059 & 4757 & & \\
\hline Bootstrapped subtotal, mean (s.d.) & $6073(897)$ & $4752(734)$ & -1321 & -3578 to 991 \\
\hline Total societal mean costs & 47010 & 37994 & & \\
\hline Bootstrapped total costs, mean (s.d.) & $46795(5630)$ & 37826 (3411) & -8969 & -21775 to 3546 \\
\hline
\end{tabular}

\begin{tabular}{|c|c|c|c|c|c|c|c|c|}
\hline \multirow{3}{*}{ Assessment } & \multicolumn{4}{|c|}{ TFP group $(n=42)$} & \multicolumn{4}{|c|}{ SFT group $(n=44)$} \\
\hline & Cost, $€$ & Utility score & BPDSI score & $\begin{array}{l}\text { Patients } \\
\text { recovered }\end{array}$ & Cost, $€$ & Utility score ${ }^{a}$ & BPDSI score & $\begin{array}{l}\text { Patients } \\
\text { recovered }\end{array}$ \\
\hline & Mean (s.d.) & Mean (s.d.) & Mean (s.d.) & $\%$ & Mean (s.d.) & Mean (s.d.) & Mean (s.d.) & $\%$ \\
\hline 1 & 4238 (3319) & $0.49(0.26)$ & $34.84(8.76)$ & 0 & 3339 (3222) & $0.46(0.31)$ & $33.14(7.13)$ & 0 \\
\hline 2 & 3712 (2702) & 049 (0.32) & $29.28(7.91)$ & 2 & 3785 (2461) & $0.49(0.30)$ & 27.43 (9.53) & 14 \\
\hline 3 & 4049 (4027) & $0.66(0.26)$ & $28.06(8.86)$ & 5 & 3549 (2057) & $0.50(0.31)$ & 24.34 (11.33) & 18 \\
\hline 4 & 3711 (2905) & $0.60(0.31)$ & $27.16(8.53)$ & 7 & 3113 (1806) & $0.46(0.35)$ & $25.31(9.58)$ & 11 \\
\hline 5 & 3413 (2502) & $0.63(0.26)$ & $24.33(10.56)$ & 19 & 2804 (1621) & $0.54(0.32)$ & $22.41(10.40)$ & 25 \\
\hline 6 & 3160 (2589) & $0.60(0.28)$ & $25.53(9.66)$ & 14 & 2536 (1479) & $0.50(0.35)$ & $21.28(11.14)$ & 34 \\
\hline 7 & 2878 (2273) & $0.63(0.29)$ & $24.96(10.81)$ & 17 & 2370 (1511) & $0.55(0.32)$ & $21.90(11.02)$ & 27 \\
\hline 8 & 2798 (2325) & $0.62(0.30)$ & 24.01 (11.03) & 19 & 2500 (1807) & $0.59(0.32)$ & $19.57(11.99)$ & 36 \\
\hline 9 & $2788(2325)$ & $0.61(0.30)$ & $22.91(11.40)$ & 31 & 2348 (1653) & $0.63(0.31)$ & $19.04(10.64)$ & 36 \\
\hline 10 & 2552 (2273) & $0.60(0.32)$ & $23.47(10.42)$ & 24 & 1961 (1402) & $0.60(0.34)$ & $19.90(11.03)$ & 36 \\
\hline 11 & 2554 (2304) & $0.60(0.31)$ & 23.01 (11.79) & 29 & 1947 (1348) & $0.61(0.32)$ & 18.01 (11.52) & 48 \\
\hline 12 & $2546(2343)$ & $0.58(0.33)$ & $24.04(11.80)$ & 26 & 1963 (1392) & $0.59(0.32)$ & $18.52(11.64)$ & 48 \\
\hline 13 & 2652 (2599) & $0.60(0.32)$ & $22.71(10.61)$ & 24 & 1946 (1617) & $0.67(0.29)$ & $17.76(11.32)$ & 45 \\
\hline $14^{\mathrm{b}}$ & $10198(10878)$ & $0.61(0.32)$ & $21.98(11.00)$ & 29 & 7172 (9912) & $0.63(0.34)$ & 16.94 (11.34) & 52 \\
\hline
\end{tabular}


Table 4 Results of main and secondary analyses

\begin{tabular}{|c|c|c|c|c|c|c|c|c|}
\hline & \multicolumn{3}{|c|}{ TFP group $(n=42)$} & \multicolumn{3}{|c|}{ SFT group ( $n=44$ ) } & \multicolumn{2}{|r|}{ ICER } \\
\hline & Costs, $€$ & $\begin{array}{l}\text { Proportion } \\
\text { of patients } \\
\text { recovered }\end{array}$ & QALYS & Costs, $€$ & $\begin{array}{l}\text { Proportion } \\
\text { of patients } \\
\text { recovered }\end{array}$ & $\begin{array}{l}\text { QALYS } \\
(n=43)\end{array}$ & $\begin{array}{l}\text { Costs per } \\
\text { recovered } \\
\text { patient }(n=86)\end{array}$ & $\begin{array}{c}\text { Costs per QALY, } € \\
(n=85)\end{array}$ \\
\hline Main analysis & 47010 & 0.29 & 2.27 & $37994^{\mathrm{a}}$ & 0.52 & 2.15 & Dominance & 90457 saving/QALY lost \\
\hline Completers analysis ( $n=53$ ) & $\begin{array}{l}42267 \\
(n=21)\end{array}$ & $\begin{array}{c}0.38 \\
(n=21)\end{array}$ & $\begin{array}{l}2.47 \\
(n=21)\end{array}$ & $\begin{array}{l}35567 \\
(n=32)\end{array}$ & $\begin{array}{c}0.59 \\
(n=32)\end{array}$ & $\begin{array}{c}2.34 \\
(n=32)\end{array}$ & Dominance & 51541 saving/QALY lost \\
\hline Baseline values for drop-outs & 49733 & 0.19 & 2.05 & $39821^{b}$ & 0.43 & 2.17 & Dominance & Dominance \\
\hline Regression corrected costs only & 28227 & 0.29 & 2.27 & 23195 & 0.52 & 2.15 & Dominance & 41933 saving/QALY lost \\
\hline $\begin{array}{l}\text { Regression corrected costs } \\
\text { and QALYS }\end{array}$ & 28227 & NA & 1.97 & $\begin{array}{l}22115 \\
(n=43)\end{array}$ & NA & 1.87 & NA & 64008 saving/QALY lost \\
\hline $\begin{array}{l}\text { Regression corrected costs } \\
\text { and BPDSI score }\end{array}$ & 28227 & 0.67 & NA & 23195 & 0.82 & NA & Dominance & NA \\
\hline Healthcare perspective & 20512 & 0.29 & NA & 18969 & 0.52 & NA & Dominance & NA \\
\hline Half unit-price session & 41572 & & & 31521 & & & & \\
\hline Double unit-price session & 57886 & & & 50939 & & & & \\
\hline Half unit-price informal care & 42899 & & & 36467 & & & & \\
\hline Double unit-price informal care & 55232 & & & 41047 & & & & \\
\hline Half out-of-pocket costs & 45599 & & & 37017 & & & & \\
\hline Double out-of-pocket costs & 49831 & & & 39947 & & & & \\
\hline
\end{tabular}

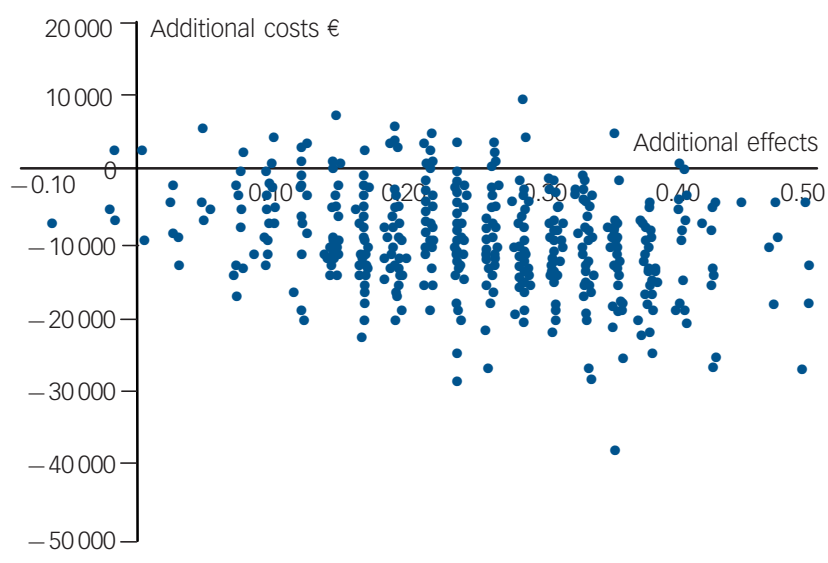

Fig. 1 Bootstrapped costs and effects for cost per recovered patient, showing 1000 bootstrap replications for incremental cost-effectiveness of schema-focused therapy compared with transference-focused psychotherapy.

Costs are plotted on the $y$ axis and effects on the $x$ axis, so a bootstrap replication in the south-east quadrant means that schema-focused therapy is less costly and more effective than transference-focused psychotherapy for that replication. and less effective. The south-east quadrant (i.e. the dominance quadrant) contains $28 \%$ of the replications, and the final $4 \%$ are located in the inferiority quadrant. The cost-effectiveness acceptability curve (Fig. 4, main analysis) indicates that the probability of SFT being cost-effective decreases with an increasing threshold value. Assuming that society's maximum willingness to pay is $€ 20000$ for a QALY gain (or accepts a minimum compensation of $€ 20000$ for a QALY loss), the probability that SFT is cost-effective is $84 \%$.

\section{Secondary analyses}

\section{costs per recovered patient}

Table 4 also shows the secondary analyses. For costs per recovered patient, results were robust, meaning that SFT dominates TFP. The cost-effectiveness acceptability curves demonstrate that, regardless of the threshold value, the probability that SFT is cost-effective is consistently over $90 \%$ (Fig. 2). From a healthcare perspective, the CEAC is less favourable for SFT. With respect to varying the unit prices and the out-of-pocket costs, total costs of SFT stayed below costs of TFP in all cases.

\section{Costs per QALY}

With respect to costs per QALY (Table 4), results were robust when using the completers analysis and the regression corrected analyses. However, using the baseline values for imputation of missing values, SFT became dominant. The cost-effectiveness acceptability curves (Fig. 4) show that baseline value imputation leads to more favourable results for SFT compared with the main analysis. However, regression correction and a completers analysis both lead to less favourable results for SFT. The descending CEACs mean that the probability of SFT being cost-effective decreases if the value society attaches to a QALY increases, dropping from $96 \%$ (best case) to $53 \%$ (worst case).

\section{Discussion}

Schema-focused therapy, compared with TFP, was cost-effective when clinical outcome was considered. However, results in terms of QALYs were uncertain. Total societal costs were not statistically significantly different between treatments, although costs for TFP were almost $€ 9000$ higher than for SFT; only the costs of informal care were significantly higher for the former therapy. This might be a consequence of the fact that in SFT professional support is a central part of therapy, offering patients the possibility of 


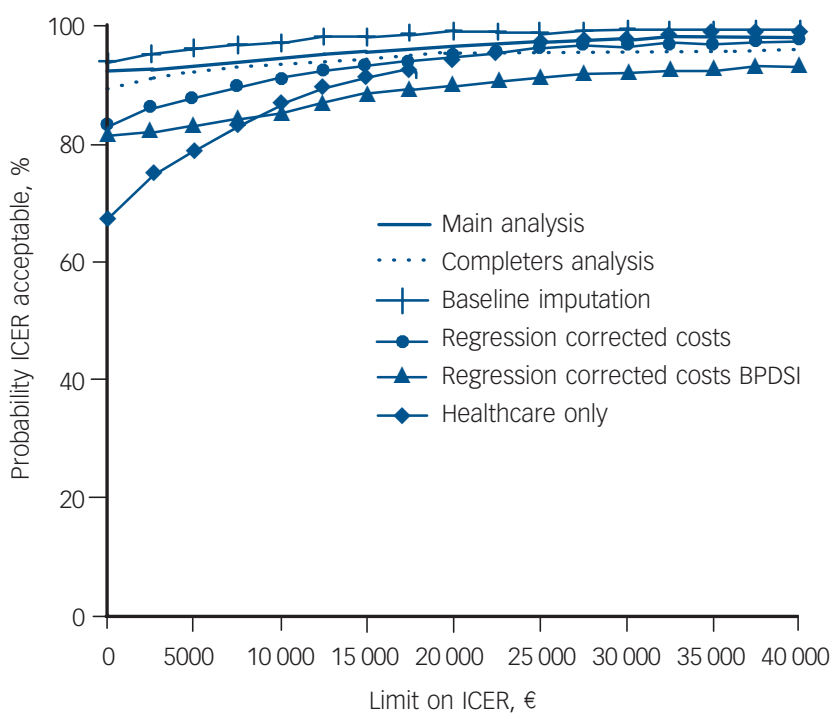

Fig. 2 Cost-effectiveness acceptability curves for secondary analyses of cost per recovered patient.

BPDSI, Borderline Personality Disorder Severity Index; ICER, incremental costeffectiveness ratio. The curves indicate the probability ( $y$ axis) of schema-focused therapy being cost-effective compared with transference-focused psychotherapy, given the threshold value ( $x$ axis) for recovering a patient.

telephone contacts and extra sessions with their therapist, whereas TFP may be more reliant on informal care. The fact that the larger part of the total costs in both therapies was outside the healthcare sector demonstrates the importance of using a societal viewpoint in economic evaluations of interventions for borderline personality disorder. Although direct healthcare costs were not statistically significantly different, there were notable differences between both groups. For the SFT group costs of psychotherapy and telephone contacts were higher, whereas for the TFP group the costs of general practitioner care, mental healthcare and other healthcare facilities were higher. Again, it can be speculated that these differences might be explained by the fact that patients found more comfort and support in SFT, whereas in TFP this was additionally sought in other healthcare facilities.

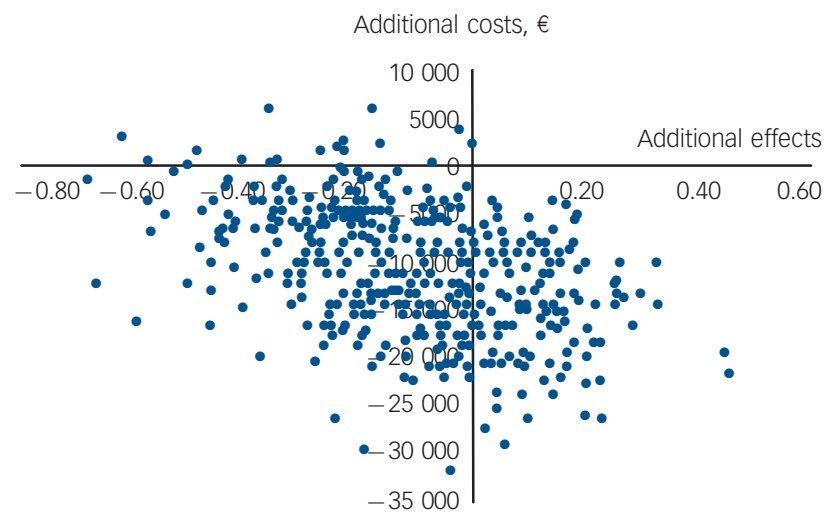

Fig. 3 Bootstrapped costs and effects for cost per quality adjusted life-year, showing 1000 bootstrap replications for incremental cost-effectiveness of schema-focused therapy compared with transference-focused therapy.

Costs are plotted on the $y$ axis and effects on the $x$ axis, so a replication in the south-west quadrant means that schema-focused therapy is less costly but also less effective than transference-focused psychotherapy for that replication.

\section{QALYS}

Utility scores increased from 0.49 to 0.61 in the TFP group and from 0.46 to 0.63 in the SFT group, improvements of 0.12 and 0.17 respectively. The minimally important difference for EQ$5 \mathrm{D}$ utility scores is considered to be 0.03 points; ${ }^{20}$ both therapies thus had a clinically relevant effect on quality of life. Therefore, in this sample, the EQ-5D can be considered sensitive to change. However, the resulting QALY was unable to discriminate significantly between the two intervention therapies, although the proportion of recovered patients was higher in the SFT group. Quality-adjusted life-years based on the 4-year period were slightly lower for the SFT group, resulting in an ICER indicating a saving of over $€ 90000$ for a QALY loss.

To date there is no consensus about a reasonable threshold value for cost-effectiveness. Most studies with the explicit goal of determining a threshold found values ranging from $€ 10000$ to $€ 27000 .^{21-23}$ However, in practice, thresholds used for appraisal of new interventions may be higher, around $€ 40000$ for the UK and $€ 80000$ for The Netherlands. ${ }^{24,25}$ The CEACs show a range of thresholds, varying from $€ 0$ to $€ 40000$. In this study, when applying a $€ 20000$ per QALY threshold, SFT in the main analysis would have an $84 \%$ probability of being cost-effective. In the secondary analyses, the worst-case scenario had a probability of $68 \%$ and the best-case scenario had a probability of $94 \%$. Given this range, the probability that SFT is more cost-effective than TFP in terms of QALYs can be considered moderate to high, in addition to the fact that this probability was already high for the analysis per recovered patient.

\section{BPDSI v. EQ-5D}

It is difficult to explain what the reason for the divergence between BPDSI and QALY results might be. When a patient recovers from borderline personality disorder, one would expect the HRQoL to improve correspondingly to at least some extent. In the long run, this was indeed the case: the slopes in increase of HRQoL were significantly steeper in the SFT group than in the TFP group. ${ }^{3}$ However, during the first half-year of treatment, QALY and BPDSI scores diverged markedly, and as a consequence, during the first 2 years of treatment, the HRQoL in the SFT patients was lower than that in TFP patients. This difference is difficult to understand as it was not paralleled in any of the clinical outcome measures. At follow-up at 4 years, the HRQoL in the SFT group was slightly higher than the HRQoL in the TFP group, but not enough to offset the total QALY difference. Maybe SFT is initially experienced as more burdensome by patients, and it may take time for clinical improvement to be translated into an increase in HRQoL. Another reason for the discrepancy might be a difference in responsiveness between the EQ-5D, which has 5 items each with three-point scale responses, and the BPDSI, which has 70 items with mostly ten-point scale responses. There is little published information concerning the use of the EQ-5D in a population with borderline personality disorder. For depression, the EQ-5D seems useful and sensitive to change, ${ }^{26,27}$ in schizophrenia, results are contradictory. ${ }^{28,29}$

\section{Limitations}

A number of limitations should be addressed. First, we used the last observation carried forward method to impute missing assessments. Although this is in accordance with the clinical study, this method is regarded as naïve. ${ }^{30}$ We therefore also performed a multiple imputation. ${ }^{31}$ However, probably because of the high cumulative drop-out at the last three measurements, point 


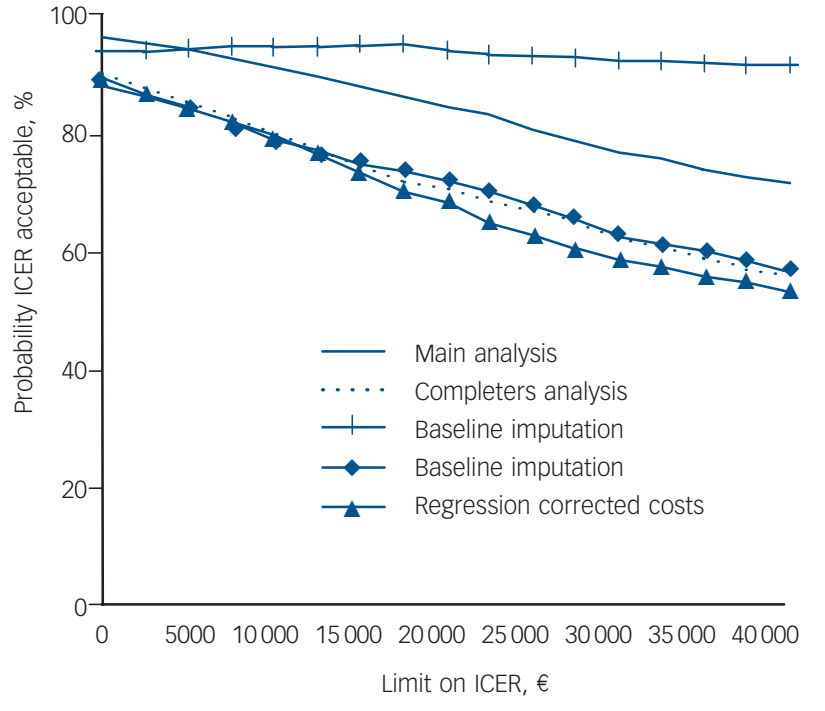

Fig. 4 Cost-effectiveness acceptability curves for secondary analyses of cost per quality-adjusted life-year.

ICER, Incremental cost-effectiveness ratio. The curves indicate the probability ( $y$ axis) of schema-focused therapy being cost-effective compared with transference-focused psychotherapy, given the threshold value ( $x$ axis) for a quality-adjusted life-year.
QALYs, the probability that SFT is cost-effective is moderate to high.

Future research should further investigate the reason for the discrepancy between the BPDSI and QALY scores, and the relation between the EQ-5D and other measures of quality of life among people with borderline personality disorder. Furthermore, we agree with Giesen-Bloo et al that different comparators such as care as usual and natural course should be studied, ${ }^{3}$ including economic evaluations with a longer period of follow-up.

Antoinette D. I. van Asselt, Carmen D. Dirksen, Department of Clinica Epidemiology and Medical Technology Assessment, University Hospital Maastricht, Maastricht; Arnoud Arntz, Department of Medical, Clinical and Experimental Psychology, Maastricht University; Josephine H. Giesen-Bloo, Department of Medical, Clinical and Experimental Psychology, Maastricht University, and Department of Medical Psychology, University Hospital Maastricht, Maastricht; Richard van Dyck of Medical Psychology, University Hospital Maastricht, Maastricht; Richard van Dyck, Spinhoven, Department of Clinical and Health Psychology and Department of Psychiatry, Leiden University, Leiden; Willem van Tilburg, Department of Psychiatry, VU Medical Center/GGZ BuitenAmstel, Amsterdam; Ismay P. Kremers, Department of Clinical and Health Psychology and Department of Psychiatry, Leiden University, Leiden; Marjon Nadort, Department of Psychiatry, Vu Medical Center/GGZ

BuitenAmstel, Amsterdam; Johan L. Severens, Department of Clinical Epidemiology and Medical Technology Assessment, University Hospital Maastricht, and Department of Health Organisation, Policy and Economics, Care and Public Health Research Institute, Maastricht University, Maastricht, The Netherlands

Correspondence: Thea van Asselt, University Hospital Maastricht, Department KEMTA, PO Box 5800, 6202 AZ Maastricht, The Netherlands. Email: avas@kemta.azm.nl

First received 21 Nov 2006, final revision 23 Oct 2007, accepted 6 Dec 2007

\section{Acknowledgements}

estimates resulting from the multiple imputation tended heavily towards extreme (and even impossible) values. Because this tendency could not be justified from the trends in the observed data, we have not included the multiple imputation results in this paper. Second, the fact that we only included disorder-related costs might have led to bias in the estimate of the total costs, since patient judgement of what is and what is not disorder-related may be flawed. However, as we do not expect patients' judgements in the SFT group to differ systematically from those in the TFP group, this presumably did not affect the incremental costs. Third, the time horizon of the analysis was 4 years; more long-term information should be collected to confirm the results now presented. Fourth, the economic evaluation was limited to the comparison of only two possible treatments. Other alternatives, such as natural course and care as usual, have not been considered. The use of a single randomised controlled trial as a vehicle for economic evaluation is often an insufficient basis for decisionmaking, because decision-makers need to be informed about costs and effects for the full range of alternative interventions. ${ }^{32}$ In that respect, our study has contributed evidence to the broader case of cost-effectiveness of treatment for borderline personality disorder.

\section{Implications of the study}

As this is the first cost-effectiveness analysis of psychotherapy in the field of borderline personality disorder from a comprehensive societal perspective, it is difficult to place the results in a broader perspective. Even for the much better-documented case of treatment for depression, comparisons between studies are complicated because of vastly different interventions, outcome measures and cost perspectives. ${ }^{33}$ The same applies to personality disorders in general and borderline personality disorder in particular. However, our study shows that long-term psychotherapy for borderline personality disorder improves HRQoL and decreases societal costs. In terms of recovered patients, there is a high probability that SFT is more cost-effective than TFP. In terms of
We are grateful to all patients, research assistants and therapists who took part in this trial. We thank Fons Kessels for his support in performing the statistical analyses. The clinical trial and the economic evaluation were financially supported by the Dutch Healthcare Insurance Board (CVZ grant OG 97-002).

\section{References}

1 American Psychiatric Association. Diagnostic and Statistical Manual of Mental Disorders (4th edn) (DSM-IV). American Psychiatric Association, 1994.

2 Ten Have M, Lorsheyd J, Bijl R, Osterthun P. Annual Report Mental Health Care [in Dutch]. De Tijdstroom, 1995.

3 Giesen-Bloo J, van Dyck R, Spinhoven P, van Tilburg W, Dirksen C, van Asselt T, Kremers I, Nadort M, Arntz A. Outpatient psychotherapy for borderline personality disorder: randomized trial of schema-focused therapy vs transference-focused psychotherapy. Arch Gen Psychiatry 2006; 63: 649-58.

4 Linehan MM, Armstrong HE, Suarez A, Allmon D, Heard HL. Cognitivebehavioral treatment of chronically parasuicidal borderline patients. Arch Gen Psychiatry 1991; 48: 1060-4.

5 Van Asselt ADI, Dirksen CD, Arntz A, Severens JL. The cost of borderline personality disorder: societal cost of illness in BPD-patients. Eur Psychiatry 2007; 22: 354-61.

6 Goossens ME, Rutten-van Molken MP, Vlaeyen JW, van der Linden SM. The cost diary: a method to measure direct and indirect costs in costeffectiveness research. J Clin Epidemiol 2000; 53: 688-95.

7 Van den Brink M, van den Hout WB, Stiggelbout AM, Putter $H$, van de Velde CJ, Kievit J. Self-reports of health-care utilization: diary or questionnaire? Int J Technol Assess Health Care 2005; 21: 298-304.

8 Severens JL, Mulder J, Laheij RJ, Verbeek AL. Precision and accuracy in measuring absence from work as a basis for calculating productivity costs in The Netherlands. Soc Sci Med 2000; 51: 243-9.

9 Van den Berg B, Brouwer WB, Koopmanschap MA. Economic valuation of 2004; 5: 36-45.

10 Oostenbrink JB, Bouwmans CAM, Koopmanschap MA, Rutten FF. Manual for cost Research. Methods and Unit Prices for Economic Evaluations in Health Care [in Dutch]. College voor Zorgverzekeringen, 2004.

11 Dutch Health Insurance Board. Pharmacotherapeutic Compass [in Dutch] Amstelveen, 2000.

12 Rice DP, Cooper BS. The economic value of human life. Am J Public Health Nations Health 1967; 57: 1954-66. informal care. An overview of methods and applications. Eur $J$ Health Econ 
13 Arntz A, van den Hoorn M, Cornelis J, Verheul R, van den Bosch WM, de Bie AJ. Reliability and validity of the borderline personality disorder severity index. J Personal Disord 2003; 17: 45-59.

14 Brooks R. EuroQol: the current state of play. Health Policy 1996; 37: 53-72.

15 Dolan P. Modeling valuations for EuroQol health states. Med Care 1997; 35 1095-108.

16 Briggs AH, Wonderling DE, Mooney CZ. Pulling cost-effectiveness analysis up by its bootstraps: a non-parametric approach to confidence interval estimation. Health Econ 1997; 6: 327-40.

17 Efron B, Tibshirani RJ. An Introduction to the Bootstrap. Chapman \& Hall 1993.

18 Van Hout BA, Al MJ, Gordon GS, Rutten FF. Costs, effects and C/E-ratios alongside a clinical trial. Health Econ 1994; 3: 309-19.

19 Manca A, Hawkins N, Sculpher MJ. Estimating mean QALYs in trial-based cost-effectiveness analysis: the importance of controlling for baseline utility Health Econ 2005; 14: 487-96.

20 Marra CA, Woolcott JC, Kopec JA, Shojania K, Offer R, Brazier JE, Esdaile JM Anis AH. A comparison of generic, indirect utility measures (the HUI2, HUI3, SF-6D, and the EQ-5D) and disease-specific instruments (the RAQOL and the HAQ) in rheumatoid arthritis. Soc Sci Med 2005; 60: 1571-82.

21 Gyrd-Hansen D. Willingness to pay for a QALY. Health Econ 2003; 12: 1049-60.

22 King JT, Tsevat J, Lave JR, Roberts MS. Willingness to pay for a qualityadjusted life year: implications for societal health care resource allocation. Med Decis Making 2005; 25: 667-77.

23 Laupacis A, Feeny D, Detsky AS, Tugwell PX. How attractive does a new technology have to be to warrant adoption and utilization? Tentative guidelines for using clinical and economic evaluations. CMAJ 1992; 146: 473-81.
24 Devlin N, Parkin D. Does NICE have a cost-effectiveness threshold and what other factors influence its decisions? A binary choice analysis. Health Econ 2004: 13: 437-52.

25 Council for Public Health and Health Care. Sensible and Sustainable Care [in Dutch]. Den Haag, 2006

26 Hayhurst H, Palmer S, Abbott R, Johnson T, Scott J. Measuring health-related quality of life in bipolar disorder: relationship of the EuroQol (EQ-5D) to condition-specific measures. Qual Life Res 2006; 15: 1271-80.

27 Sapin C, Fantino B, Nowicki ML, Kind P. Usefulness of EQ-5D in assessing health status in primary care patients with major depressive disorder. Health Qual Life Outcomes 2004; 2: 20.

28 Van de Willige G, Wiersma D, Nienhuis FJ, Jenner JA. Changes in quality of life in chronic psychiatric patients: a comparison between EuroQol (EQ-5D) and WHOQoL. Qual Life Res 2005; 14: 441-51.

29. Prieto L, Sacristan JA, Hormaechea JA, Casado A, Badia X, Gomez JC. Psychometric validation of a generic health-related quality of life measure (EQ-5D) in a sample of schizophrenic patients. Curr Med Res Opin 2004; 20 $827-35$

30 Oostenbrink JB, Al MJ. The analysis of incomplete cost data due to dropout. Health Econ 2005; 14: 763-76.

31 Rubin DB, Schenker N. Multiple imputation in health-care databases: an overview and some applications. Stat Med 1992: 10: 585-98.

32 Sculpher MJ, Claxton K, Drummond M, McCabe C. Whither trial-based economic evaluation for health care decision making? Health Econ 2006; 15 $677-87$

33 Barrett B, Byford S, Knapp M. Evidence of cost-effective treatments for depression: a systematic review. J Affect Disord 2005; 84: 1-13.

\section{Tony Maden}

Even worse than asking a silly question is paying lawyers to answer it for you. Treatability was never a yes/no matter and all we have to show for years of medico-legal argument is an expensive 'maybe' and the absurd suggestion that sitting in hospital is treatment. Meanwhile other doctors quietly dropped the notion of 'incurables' long ago. So let the lawyers and independent experts earn their money elsewhere. Personality disorder is a psychological disability. Quick or simple remedies are no more likely than for chronic schizophrenia - and the principles of rehabilitation are also the same. So get on with it. 